\title{
Healthcare Information Technology Infrastructures in Turkey
}

\author{
A. Dogac' ${ }^{1}$ M. Yuksel', G. L. Ertürkmen', Y. Kabak', T. Namli', M. H. Yıldız', Y. Ay², B. Ceyhan³, \\ Ü. Hülür ${ }^{3}$, H. Öztürk ${ }^{3}$, E. Atbakan ${ }^{3}$ \\ I SRDC Ltd., Ankara, Turkey \\ 2 Sürat Teknoloii, Ankara, Turkey \\ ${ }^{3}$ Ministry of Health, Ankara, Turkey
}

\section{Introduction and Objectives}

The Health Transformation Program [1] of the Ministry of Health, Turkey, was published in 2003. One of the main components of this program is achieving e-health, with the following objectives:

- Ensuring standardization of data used in healthcare

- Creating the Electronic Health Record for citizens

- Data analysis support for managers (Decision Support System)

- Speeding up the flow of information among stakeholders

- Saving resources and increasing efficiency in the healthcare system.

Following the Health Transformation Program, several healthcare IT infrastructures have been developed in Turkey. The objective of this paper is to describe some of them including Sağlik-Net, together with its two major components, namely, the National Health Information System (NHIS) [2] and the Family Medicine Information System (FMIS) [3]. The other infrastructures surveyed include the Centralized Hospital Appointment System (CHAS) [4], the Basic Health Statistics Module (BHSM), the Core Resources Management System (CRMS) and the e-prescription system of the Social Security Institution. Figure 1 shows the overall architecture of the Sağlik-Net. In addition to the major components, it also contains the National Health Data Dictionary (NHDD) [5], the Health Coding Reference Server (HCRS) [6] and the Decision Support Systems. The Sağlik-Net is also integrated with the national e-prescription infrastructure maintained by the Social Security Institution. The Turkish e-identity pilot project has successfully been concluded and distribution of e-identity cards to Turkish citizens will start by January 2014 .

The paper is organized as follows: Section 2 briefly summarizes the Health Coding Reference Server and Section 3 describes the National Health Data Dictionary. Section 4 presents NHIS which has already been described in detail in [2] and briefly explained here for the sake of completeness by also stressing the updates in the version released in 2012. Section 5 describes the Family Medicine Information System. Section 6 presents the Centralized Hospital Appointment System. Basic Health Statistics Module is summarized in Section 7 and Core Resources Management System in Section 8. Section 9 presents the e-prescription infrastructure. The international collaboration projects of Turkey which are integrated to Sağlik-Net are given in Section 10. Finally, Section 11 concludes the article.

\section{The Health Coding Reference Server}

The Health Coding Reference Server encapsulates all the international and national coding systems used in Turkey within a publicly accessible server.

Some of the coding systems available from Health Coding Reference Server are international such as ICD-10 [7] or Anatomical Therapeutic Chemical Classification System [8] and most are locally defined for certain sets of information such as Clinics, Patient Discharge Type, Pregnancy Result, or Baby Monitoring Calendar. Currently, there are 329 coding systems maintained in the server.

\section{The National Health Data Dictionary}

The National Health Data Dictionary [5] contains the commonly used healthcare 


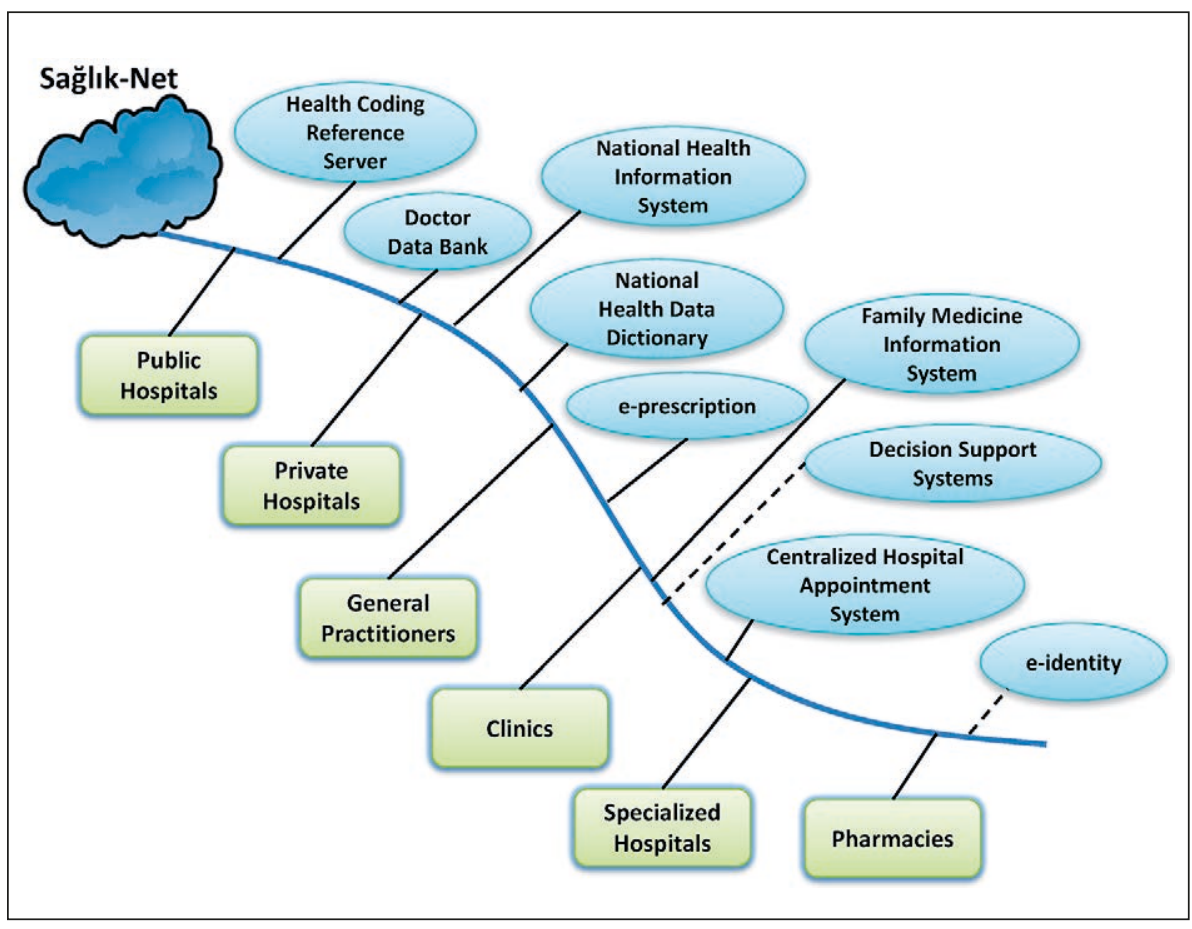

Fig. 1 Sağlık-Net

data elements such as "Address", "Name", "Main Diagnosis", "Vaccination", and "Treatment Method". The format of these data elements is defined according to the rules and guidelines given in ISO/IEC 11179-4 Standard [9]. The first version of the dictionary that was active from 2008 to mid 2012 had 261 data elements, while the current version, which is an update of the first version based on feedback from the users and decision makers, has 464 data elements.

The "Aggregate Core Components", which are called Minimum Health Data Sets (MHDSs), are formed using these data elements [2]. The Minimum Health Data Sets define the data that emerge at the time of presenting a specific healthcare service, e.g. infant monitoring data set or pregnant monitoring data set. There were 46 Minimum Health Data Sets in the first version of the National Health Data Dictionary, and now there are 65 of them.

The data elements within the Minimum Health Data Sets are mostly coded using coding systems that are available from the Health Coding Reference Server.

\section{National Health Information System (NHIS)}

The National Health Information System of Turkey (NHIS) [2] is a nation-wide infrastructure for collecting and to some extent sharing patients' Electronic Health Records (EHRs). The current implementation of NHIS supports the transfer of episodic EHRs from secondary and tertiary healthcare provider information systems to NHIS servers at the Ministry of Health. Yet, only the general practitioners (GPs) can access the EHRs of their own patients, through their Family Medicine Information System (FMIS) client applications.

In the first version of the NHIS, which became operational in January 2009, NHIS and FMIS were two separate systems with their own databases and Web services on the server side, which created problems in linking the EHR data between primary care and secondary/tertiary care. This problem is solved in August 2012 with the 2.0 release of the NHIS, which is an improved, standards compliant and technically more capable redevelopment of the first release, based on the experiences gained from its first version. In the new release, the data collected through the previous NHIS and FMIS systems are merged and preserved. With NHIS 2.0, all the client side applications of primary, secondary and tertiary care providers interact with the unique national system.

The episodic EHRs collected through the NHIS, also called the Transmission Data Sets, are aggregated from the Minimum Health Data Sets. In the previous version of NHIS implementation, there were 41 Transmission Data Sets; each dedicated to a Minimum Health Data Set such as "15-49 Age Female Observation", "Mouth and Teeth Examination", "Vaccine Notification", "Infant Nutrition" and "Diabetes". In the current release of NHIS, there are only seven broader Transmission Data Sets, namely "Citizen Registration", "Examination", "Patient Demographics", "Test Results", "Inpatient", "HIV" and "Death Notification", which are capable of collecting all 65 Minimum Health Data Sets in various combinations.

The Transmission Data Sets are mapped to HL7 CDA Release 2 to create the "Transmission Schemas" as described in [2]. In brief, the Minimum Health Data Sets are mapped to CDA sections, and the data elements of the MHDSs are mapped to CDA entry classes and their attributes. A "Transmission Schema" instance constitutes the payload of an NHIS EHR exchange message and HL 7 v3 Web Services Profile [10] is used at the transport layer.

It should be noted that the data required by the NHIS is automatically generated by the healthcare provider information systems and are sent by invoking NHIS Web Services; hence the process is transparent to the health professionals who create the content of the EHR documents. The health professionals continue using the healthcare provider information systems as before; the vendors of these systems have developed wrapper applications that populate the NHIS conformant messages from the data collected from their own systems and send them by invoking the NHIS Web Services.

NHIS is open to extensions: When a need for a new EHR document arises, the existing Minimum Health Data Sets are re-used if possible; if not, the new Minimum Health Data Sets are constructed by using the ex- 
isting data elements and the National Health Data Dictionary is expanded by defining new Data Elements when necessary. These updates are then reflected to the operational electronic services. So far, one major and a number of minor updates have taken place.

\section{Family Medicine Information System (FMIS)}

In comparison to many other countries, Turkey was late in migrating to the family medicine practice. Previously, primary healthcare was delivered either through health posts distributed all over the country or by hospitals which are conventionally responsible for the secondary and tertiary healthcare services. However, with the introduction of Health Transformation Program in 2003, there has been considerable progress in realizing family medicine in Turkey.

\subsection{Implementation of FMIS}

The Family Medicine Information System is a national system for exchanging primary care records among general practitioners and the Ministry of Health. The content of healthcare data under the GPs responsibility is also defined through the Minimum Health Data Sets, which are available in the National Health Data Dictionary.

The FMIS has a client-server based architecture. The centralized part is hosted by the Ministry of Health in Ankara and the desktop client applications are used by the GPs in their own offices. The client applications can both store into and retrieve data from the Ministry servers. Communication is based on Web Services exposed on the Ministry side. Prior to August 2012, FMIS had its dedicated central database and proprietary Web Services for communicating with the FMIS client applications. With NHIS 2.0, these client applications use the HL7 compliant Web Services of the harmonized NHIS, which are also used by secondary and tertiary healthcare providers as explained in the previous section.

Until a year ago, the Ministry of Health used to distribute a simple FMIS client ap- plication to GPs for free. Even then, many GPs opted for using more capable and user friendly FMIS client applications by $3 \mathrm{rd}$ party vendors.

The client applications that are used by the GPs can work both in online and offline modes. They can either record patient observations locally and synchronize with the MoH Web Services immediately, or synchronize the local data later. The second approach is especially useful when GPs visit their patients in the rural areas together with their laptops. As mentioned earlier, it is also possible to retrieve data from the $\mathrm{MoH}$ central servers. For example, when a citizen moves to a new city or district, the new GP assigned to him is able to retrieve all his previous records into his FMIS client application through the Web Services.

The Minimum Health Data Sets that are defined in the family medicine domain and exchanged via Web Services are (some apply to secondary and tertiary care domain as well):

- Infant Observation

- Child Observation

- Vaccine Tracking

- 15-49 Age Woman Observation

- Pregnancy Observation

- Puerperal Observation

- Death Notification

- Generic Patient Examination

- Consultation Notification

The architecture diagram of FMIS is presented in Figure 2.

\subsection{Performance Evaluation of the GPs through FMIS}

FMIS is also used to evaluate the performance of the GPs, which affect their salaries. The GPs have a baseline salary which can increase or decrease depending on their performance scores calculated by the FMIS. When they complete all the expected patient observations, their salaries increase. In the opposite case, their salaries may decrease below their baseline salaries.

The performance criteria for GPs are composed of five measures that are calculated each month:
1. Vaccine success rate: Division of the number of vaccines applied by the number of vaccines that should have been applied. The latter is calculated based on the vaccine calendar for babies who are registered to the GP. The central system keeps an instance of this vaccine calendar for each infant per GP and the success rate of a GP is calculated automatically according to the expected number versus the realized number of vaccines.

2. Infant observation success rate: Division of the number of infant observations made, by the number of infant observations that should have been made. This is similar to the vaccine success rate; this time there is an infant observation calendar which necessitates seven distinct observations until the baby is ten months old.

3. Pregnancy observation success rate: Division of the number of pregnancy observations realized by the number of pregnancy observations that should have been made. There is a pregnancy observation calendar which divides the complete pregnancy process into four periods, and in each of these periods at least one pregnancy observation has to be made by the responsible GP.

4. Referral rate: This is calculated based on the number of referrals that the GP made, the total number of patients registered to the GP and the average number of patient visits to a healthcare provider in Turkey. With this calculation, the GPs are encouraged to refer their patients to secondary healthcare services when it is necessary.

5. The number of citizens in need of mobile service: The GPs have to visit their patients who are physically impaired or living in hard to reach rural areas. These visits are within the scope of mobile services and this extra work generates extra income for the GPs.

Each GP is responsible from about 3500 citizens. When a GP is officially on leave, all of his patients are assigned to a proxy GP so that neither his patients nor he face a medically or financially disadvantaged situation. Apart from that, citizens are free to change their GPs at any time, as long as the geographic constraints are satisfied. 


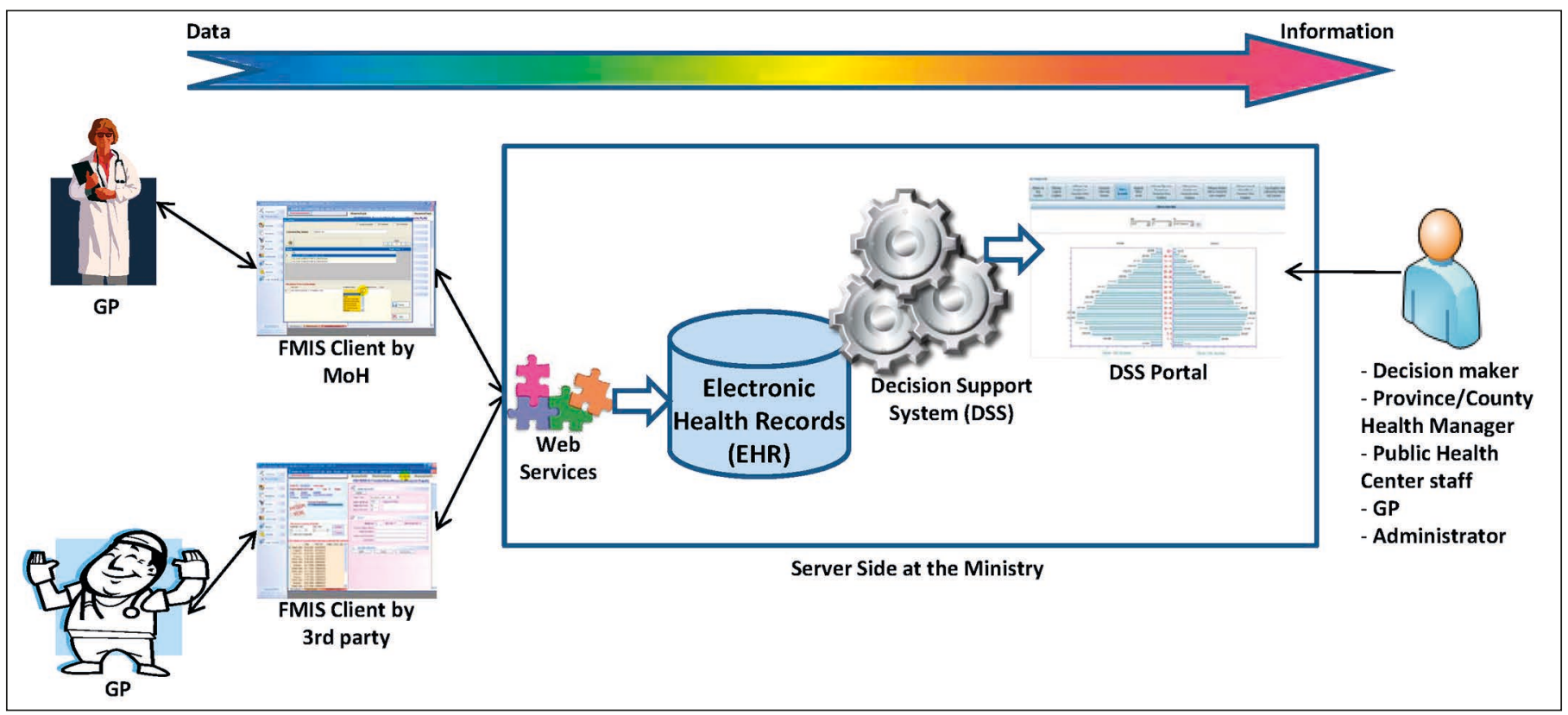

Fig. 2 FMIS Architecture

\subsection{Decision Support System of FMIS}

The Decision Support System (DSS) of FMIS provides an overall view of the primary care patient records at various levels, e.g. in the country as a whole, or statistics per province/county or per GP. Furthermore, the patient records can be grouped according to the topics while displaying statistical data. For example, with a single click, it is possible to list all women who are in the final month of their pregnancy, and then dig into their individual records, find the responsible GP, etc.

DSS is based on Oracle Business Intelligence (BI) solutions [11] and provides customized interfaces for several roles that are accessible through Web:

- The policy makers (i.e. decision makers) including the Minister of Health: They are able to see all primary care data in Turkey.

- Administrators: They can see all data and handle administrative tasks of the Web portal. They can also create new interfaces/queries.

- Province/County Health Managers: They can see all data in the province/county in which they are responsible.

- Public Health Center staff: There is a Public Health Center for each 100,000 population. The staffs of these centers have the same capabilities as the Province/County Health Managers.

- General Practitioners: The GPs are able to see all data that belong to the patients registered to them.

\section{Centralized Hospital Ap- pointment System (CHAS)}

Centralized Hospital Appointment System (CHAS) enables the citizens to make appointments in any public secondary and tertiary healthcare provider by calling the CHAS Call Center phone number "182" or online through the CHAS Web Portal [12] and mobile applications in all major mobile operating systems (i.e. Android, iOS, Windows Phone, Blackberry). The citizens are assisted by online operators during phone calls. All the healthcare professionals working in public healthcare providers share their calendars of at least 15 days with the Ministry through CHAS Web Services to enable citizens to make appointments according to these calendars. CHAS, which was introduced in 2009 in a few provinces, became operational in the whole country by 2011 .

\section{Basic Health Statistics Module (BHSM)}

Basic Health Statistics Module (BHSM) is used to collect information about the health status, health risks and indicators across the country to direct the resources and programs of Central and Provincial Directorates of the Ministry of Health. There are about 70 Basic Health Statistics Forms and some of them are reported to the World Health Organization (WHO) as well. However, most of these forms are filled out manually.

An analysis by the Ministry of Health revealed the fact that some of the data in the forms are already available from the Family Medicine Information System, such as Infant/Child Follow-up or Pregnancy Follow-up. Therefore, currently the work is in progress to improve the FMIS Decision Support System to obtain these reports automatically to replace the manual BHSM reports.

For the automation of the other forms, there are plans for the automatic generation of these reports from the National Health Information System to the extent possible. 


\section{Core Resources Manage- ment System (CRMS)}

Core Resources Management System (CRMS) is a system developed to monitor the staff, institutions, equipment of the Ministry of Health and it consists of the following components:

- Human Resources Management System (HRMS)

- Equipment Resources Management System (ERMS)

- Investment Tracing System (ITS)

- Private Health Organizations Management System (HOMS)

\subsection{Human Resources}

\section{Management System (HRMS)}

HRMS is used for monitoring the staff information and activities including payroll and accrual processing. It covers the following applications: Appointment Information System, Register Information System, Staff Information System, Promotion Information System, Salary and Accrual Information System, Family Medicine Billing, Specialization/Degree Registration (Doctor Data Bank - DDB), In-service Training, Discipline Information System, Legal Regulatory Information System, HRMS Practice Management, Personnel Departments, Contracted Staff actions, 112 Emergency Medical Service Reports, National Medical Rescue Team Staff Tracking, Mobilization Automation.

This system has reached the goals of acceleration of the information flow between Ministry of Health Headquarters and 81 Provincial Directorates of Health, helping to quickly determine the needs by monitoring the sources, getting statistics and reports intended for planning, achieving the coordination and standardization between Ministry of Health Headquarters and Provincial Health Directorates.

\subsection{Equipment Resources Management System (ERMS)}

ERMS enables monitoring the equipment in stock of the all institutions under the Ministry of Health, determining the overstock equipment and following up responsibilities. It is composed of an Inventory Information System, an Equipment Check-in System, a Storage Information System, a Fixed Asset Information System, a Transportation Vehicle Information System, a Medical Device Information System, a Repairing and Maintenance Information System, and the Company Information System.

Using ERMS, it is possible to determine the overstock items in hospitals. Hospitals can query ERMS for their needs to prevent unnecessary purchases. Moreover, the system can be queried for the prices of items to compare the prices of the same or similar purchases.

The hospital information systems can also transfer data to ERMS. This communication is achieved through Web Services.

\subsection{Investment Tracing System (ITS)}

ITS is used for planning the financial resources of the Ministry of Health according to the needs, distributing the resources between the ministry units, and monitoring them. It contains the following modules: the Investment Information System and the Real-Estate Information System.

Through the Investment Information System, investment proposals, the level of construction, building repairs, construction revision processes can be monitored. With the help of the Investment Information System, it is possible to monitor the progress of a construction until it is completed.

Real-Estate Information System contains information about the buildings the Ministry owns or rents; its name, city, address, age, the result of its earthquake-resistance test, physical properties of the building, its rent, and ownership information.

\subsection{Private Health Organizations Management System (HOMS)}

Private Health Organizations Management System (HOMS) makes it possible to monitor the hardware of private health organizations, their licenses, their staff and service capacity. HOMS is integrated with the other CRMS components. For example, the registration of the doctors of a private hospital has to be done via HOMS and validation is realized automatically via CRMS.

\section{9 e-prescription System}

The Social Security Institution has developed a Web-based e-prescription infrastructure as a part of their Medula system, which was originally developed to automate the reimbursement of the medical expenses of the citizens covered by social security. After a few years of regional piloting, the e-prescription system became operational in the whole country by July 2012 .

The e-prescription system is linked with Sağllk-Net and seamlessly integrated with the healthcare provider information systems through Medula Web Services, so that the health professionals continue prescribing medications via their regular interfaces. Health professionals provide medication details together with the ICD-10 codes of patient's diagnoses in an e-prescription. Each e-prescription is assigned a unique identifier by the e-prescription system. The health professionals can also view patients' ongoing medications and the amount not yet consumed. The same facilities are offered to health professionals also through a Web-based application served by the Social Security Institution [13].

Patients apply to pharmacies for dispensation with their citizen IDs and unique e-prescription identifiers provided by the health professional. Using the Medula Pharmacy Software, the pharmacies are able to query, view and dispense the e-prescriptions. The system also keeps track of all the medications provided to the patient so far, prevents unnecessary dispensation, and offers replacements whenever a specific product is not available. Finally, the system is used by the Social Security Institution for the reimbursement of pharmacies.

\section{International Collaboration}

Ministry of Health, Turkey has been in collaboration with a number of European countries through its involvement in projects supported by the European Commission. 


\subsection{ICT-PSP epSOS: Smart Open Services for European Patients}

The aim of the epSOS Project [14] is to provide a European level cross-border interoperability platform for sharing healthcare data. Through this platform, the exchange of electronic patient summary and prescription documents among the European countries is realized. epSOS is supported by the European Commission and the health ministries of the European countries, and its consortium is composed of 48 partners from 23 European countries.

Turkey's contributions to the epSOS Project can be summarized as follows: First, Turkey provided an open source implementation of the epSOS cross-border interoperability platform. This implementation triggered an open source community with the involvement of a number of epSOS beneficiaries for further development [15]. Further contributions include participation to specification and design of some European level services such as semantic interoperability; exposing of the clinical data that are collected in Turkey's National Health Information System to Europe through standards-based epSOS interfaces; and processing of patient data that are coming from European countries. Turkey also leads the validation activities in which the conformance of country implementations to epSOS specifications and interoperability among the implementations are tested continuously.

\subsection{FP7-ICT EMPOWER: Support of Patient Empowerment by an Intelligent Self-management Pathway for Patients}

The EMPOWER Project [16] develops a modular and standards-based Patient Empowerment Framework, which facilitates the self-management of diabetes patients based on Personal Health Records (PHRs) and Self-Management Pathways through context-aware, personalized services. The services include:

- Services for the specification and execution of actions to change behavior according to diabetes-specific healthcare needs. Patients can develop personalized action plans, which include recommendations from the treating physicians and patients' preferences.

- Services for monitoring of vital, physical, mental parameters as well as physical and lifestyle activities based on health standards.

EMPOWER semantically integrates multiple information sources (EHR/PHR, diabetes guidelines, mobile devices/applications for patterns of daily living) using a shared and standards-based knowledge model. The Self-Management Pathways facilitate the specification of recommendations by the health professionals and these recommendations allow specifying individual goals for the patients.

The pilot applications are being realized in Germany and in Turkey to demonstrate that the patient-centric approach of EMPOWER can improve disease management by personalized self-management services helping diabetes patients to cope better with their diseases. For this purpose, through its standards-based interfaces, the National Health Information System is already integrated with the EMPOWER Patient Empowerment Framework within the scope of Turkish pilot.

\subsection{ICT-PSP PALANTE: PAtient Leading and mANaging their healThcare through Ehealth}

The PALANTE Project [17] aims to empower patients to lead and manage their healthcare by informing the patients about their health problems with the help of a Personal Health Record (PHR) system. It also supports managing chronic diseases, and helps to reduce costs with the help of tele-medicine.

There are seven pilot applications in six different countries. The Turkish pilot focuses on patients suffering from severe arthritis. The Virtual Arthritis Clinic Service of the pilot is a Web based patient-doctor shared arthritis disease management system, which is already integrated with both the National Health Information System and the local hospital information systems of hospitals in two different pilot regions.
The end-users (both health professionals and patients) provided by the Turkish Ministry of Health are involved in the pilot from the beginning of the project, in order to guide the requirement analysis and design phases.

\section{Conclusions}

This article surveys some of the major healthcare IT infrastructures which are realized as a part of the Health Transformation Program of Turkey [1] that began in 2003.

One of the main healthcare IT infrastructures, the NHIS, became operational in January 2009, which was then updated in August 2012 based on the experiences and lessons learned during the operation of the first release. In its first release, NHIS and FMIS were two different infrastructures and attempts to merge them failed several times because; i) these two similar but disconnected systems had different data and connection interfaces for collecting the same medical data, and ii) they were being used heavily, which made it difficult to interrupt one for a period of time for the merging operation. The second release of NHIS implemented a single EHR system to serve for all primary, secondary and tertiary care integrating (former) NHIS and FMIS data. Another handicap of the first release of NHIS was that the transmission schemas were HL7 v3 Web Services compliant and reused HL7 CDA R2 schema; however they were not totally CDA compliant due to renaming of attributes as previously explained in detail in [18]. Although this was done to facilitate the interpretation of the Web Service schemas by the developers; in the long run, it caused maintainability problems for both the Ministry and the vendors: Updates to schemas, even the minor ones, were difficult to achieve. In the second release of NHIS, with its full compliance to the generic CDA schema, it is possible to add new data elements or Minimum Health Data Sets to the existing Web Services with minimum effort.

By November 2013, 98\% of the public hospitals and $80 \%$ of the private and university hospitals were connected to NHIS sending the EHRs of their patients on a daily basis. The average number of EHR docu- 
ments that are sent by the healthcare provider information systems and successfully persisted in the NHIS can reach to 4.6 million a day. As of November 2013, there were more than 2 billion EHR documents in the NHIS. The number of connected healthcare providers is 3,573 , and this number is still increasing while the remaining healthcare providers are completing their integration to the NHIS. Since August 2012, all the GPs are also connected to the NHIS; hence the total number of connected nodes is 24,918 . So far, electronic healthcare records of 78.9 million people have been created in the NHIS.

Although NHIS is a successful system, currently, only the general practitioners can access the EHRs of their patients. To make EHRs accessible to the authorized health professionals in the secondary and the tertiary healthcare systems as well as the patients themselves, a legal framework and a proper patient consent mechanism are necessary. The work is going on in this direction and a pilot application is planned.

Another contribution of the Health Transformation Program of Turkey was introducing family medicine to Turkey. Family medicine plays an important role in the healthcare system by preventing inappropriate referrals that may cause loss of time for individuals and service providers, irregularities and unnecessary health expenditures. Currently family medicine is available in all 81 provinces of Turkey supported by FMIS. As of November 2013, there were 21,345 GPs on duty in Turkey and all were connected to the FMIS.

Centralized Hospital Appointment System enables the citizens to make appointments in any public secondary and tertiary healthcare provider together with Mouth and Teeth Health Centers by calling the CHAS Call Center or online through the CHAS Web Portal and mobile applications. 111 million appointments have been made via CHAS so far. As an example to the daily usage, in the second week of November 2013 the average number of appointments a day was 281 thousand and the maximum number of appointments a day was 331 thousand.

Basic Health Statistics Module is a system to collect information through forms about the health status, health risks and indicators across the country to direct the resources and programs of Central and
Provincial Directorates of the Ministry of Health. However, most of these forms are filled out manually. Therefore, currently the work is in progress for the automatic generation of these reports from the NHIS to the extent possible.

The Core Resources Management System provides tracing of manpower, equipment and management of financial resources between Central Organization of Ministry of Health and agencies within 81 Provincial Health Directorates.

The e-prescription system together with Medula, the underlying medical reimbursement system of the Social Security Institution, is another successful healthcare IT infrastructure in Turkey. The system processed and reimbursed 171 million prescriptions with a total value of 7.4 billion Turkish Liras $(\sim 3.7$ billion $\$$ ) within the first six months of 2013 [19]. The e-prescription system has retired the paper based prescriptions in Turkey by seamlessly integrating with the existing information systems of the health professionals and pharmacists. It contributes to the general budget to a great extent by preventing fraudulent or unnecessary dispensations.

Finally, the Turkish authorities believe in the mutual benefits of experience sharing and open collaboration, and execute this vision through their involvement in a number of international collaborative projects. Best practices in other countries are taken into account while initiating or improving a national project.

\section{References}

1. Health Transformation Program [cited November 11, 2013]. Available from: http://www.saglik.gov.tr/TR/ belge/1-2906/saglikta-donusum-programi.html

2. Dogac A, Yuksel M, Avci A, Ceyhan B, Hulur U, Eryilmaz Z, et al. Electronic Health Record Interoperability as Realized in Turkey's National Health Information System. Methods Inf Med 2011;50(2);140-9.

3. The Family Medicine Model of Turkey [cited November 11, 2013]. Available from: http://www. ailehekimligi.gov.tr/

4. The Centralized Hospital Appointment System (CHAS) [cited November 11, 2013]. Available from (in Turkish): http://www.hastanerandevu.gov. tr/Vatandas/hakkimizda.jsp

5. The National Health Data Dictionary (NHDD) of Turkey v2.0 [cited November 11, 2013]. Available from: http://www.e-saglik.gov.tr/dosyalar/
USVS2_30032012.pdf

6. The Health Coding Reference Server (HCRS) [cited November 11, 2013]. Available from: https:// skrs3.sagliknet.saglik.gov.tr

7. International Statistical Classification of Diseases and Related Health Problems 10th Revision (ICD-10) [cited November 11, 2013]. Available from: http://apps.who.int/classifications/apps/icd/ icd10online/

8. The Anatomical Therapeutic Chemical (ATC) Classification System, WHO Collaborating Centre for Drug Statistics Methodology [cited November 11, 2013]. Available from: http://www.whocc.no/atc/

9. ISO/IEC 11179-4:2004 (Information technology -- Metadata registries (MDR) -- Part 4: Formulation of data definitions) [cited November 11, 2013]. Available from: http://www.iso.org/iso/ catalogue_detail.htm?csnumber $=35346$

10. HL7 Web Services Profile, Release 2 [cited November 11, 2013]. Available from: http://www.hl7.org/ special/committees/projman/searchableprojectindex. cfm?action=edit\&ProjectNumber=30111.

11. Oracle Business Intelligence (BI) solutions [cited November 11, 2013]. Available from: http:// www.oracle.com/us/solutions/business-analytics/ business-intelligence/overview/index.html

12. The Citizen Portal of the Centralized Hospital Appointment System [cited November 11, 2013]. Available from: http://www.mhrs.gov.tr/Vatandas/

13. The Web interface of the e-prescription system for health professionals [cited November 11, 2013]. Available from: https://medeczane.sgk.gov.tr/doktor

14. The epSOS Project: Smart Open Services for European Patients [cited November 11, 2013]. Available from: http://www.epsos.eu

15. OpenNCP Community [cited November 11, 2013]. Available from: https://openncp.atlassian.net/wiki/

16. The EMPOWER Project: Support of Patient Empowerment by an intelligent self-management pathway for patients [cited November 11, 2013]. Available from: http://www.empower-fp7.eu

17. The PALANTE Project: PAtient Leading and mANaging their healThcare through Ehealth [cited November 11, 2013]. Available from: http://www. palante-project.eu

18. Kabak Y, Dogac A, Kose I, Akpinar N, Gurel M, Arslan Y, et al. The Use of HL7 CDA in the National Health Information System (NHIS) of Turkey. 9th International HL7 Interoperability Conference (IHIC), Crete, Greece, October 2008. p. 49-55.

19. Monthly Basic Indicators - July 2013, Social Security Institution [cited November 11, 2013]. Available from: http://www.sgk.gov.tr/wps/wcm/ connect/286dd8f7-64d9-4c5f-8774-3632ce6b164c/2013_temmuz_sosyalguvenlik_temelgostergeleri.pdf

\section{Correspondence to:}

Dr. Asuman Dogac

SRDC Software Research, Development and Consultation Ltd. ODTU Silikon Bina, 1. Kat, №:14

Middle East Technical University Campus

06800 Ankara, Turkey

Phone: +90 (312) 2101393

Fax: +90 (312) 2101837

E-mail: asuman@srdc.com.tr 